\title{
Double-Balloon Endoscopy after Incomplete Colonoscopy and Its Comparison with Computed Tomography Colonography
}

\author{
Carlijn Hermans ${ }^{1}$, Dennis van der Zee ${ }^{2}$ and Lennard Gilissen ${ }^{1}$ \\ Departments of ${ }^{1}$ Gastroenterology and Hepatology, ${ }^{2}$ Radiology, Catharina Hospital Eindhoven, Eindhoven, Netherlands
}

Background/Aims: Because of the national screening program for colorectal carcinoma in The Netherlands, the number of colonoscopies has increased. In case of incomplete colonoscopy, computed tomography colonography (CTC) and double-balloon colonoscopy $(\mathrm{DBc})$ are alternative options. This study evaluated cecal intubation rate and pathology detection rate in the previously unexplored part of the colon, complication rate of $\mathrm{DBc}$, and CTC results after incomplete colonoscopy.

Methods: Retrospective observational study in a tertiary referral hospital regarding DBc and CTC reports from cases with incomplete colonoscopy.

Results: Sixty-three DBcs were performed after incomplete colonoscopy. Cecal intubation rate was 95\%. Detection rate was 58\% (5\% carcinoma and 3\% high-grade dysplastic adenoma). CTC preceded $54 \%$ of DBcs and $62 \%$ of CTC findings were confirmed. In $16 \%$, a biopsy was taken, and in $60 \%$, an intervention (mostly polypectomy) was performed. One major complication (1.5\%) occurred, i.e., arterial bleeding due to polypectomy necessitating right hemicolectomy. CTC $(n=213)$ showed a possible lesion in $35 \%$, and could be confirmed by follow-up endoscopy or surgery in $65 \%$.

Conclusions: $\mathrm{DBc}$ is effective and safe for completion of colon inspection in incomplete colonoscopy. In patients with a high likelihood of pathology, DBc is preferred over CTC. Clin Endosc 2018;51:66-71

Key Words: Double-balloon colonoscopy; Colonography, computed tomographic; Colon carcinoma; Colon visualization; Cecum

\section{INTRODUCTION}

For diagnosis and screening of colonic disease, colonoscopy is considered to be the most effective procedure, because of its diagnostic and therapeutic capability. ${ }^{1,2}$ With a rising incidence of colorectal cancer (CRC) in many parts of the world, ${ }^{3}$ population screening programs for CRC have been initiated in many countries. Colonoscopy with polypectomy has been shown to reduce both the incidence and mortality of subsequent CRC. ${ }^{4-6}$ In The Netherlands, a population screening

Received: December 28, 2016 Revised: October 8, 2017

Accepted: October 25, 2017

Correspondence: Carlijn Hermans

Department of Gastroenterology and Hepatology, Catharina Hospital Eindhoven, Michelangelolaan 2, 5623 EJ Eindhoven, Netherlands

Tel: +31-65-053-0679, Fax: +31-40-239-9751, E-mail: cjmhermans@gmail.com

(cc) This is an Open Access article distributed under the terms of the Creative Commons Attribution Non-Commercial License (http://creativecommons.org/ licenses/by-nc/3.0) which permits unrestricted non-commercial use, distribution, and reproduction in any medium, provided the original work is properly cited. program was started in 2014 in which a positive immunological fecal occult blood test is followed by colonoscopy.

Cecal intubation is the primary quality index in colonoscopy. $^{2}$ Even experienced endoscopists fail to reach the cecum in $2 \%-10 \%$ of colonoscopies, ${ }^{7-9}$ because of dolichocolon, loop formation, and angulation of the colon. ${ }^{9-14}$ Previous literature has shown that several factors have been associated with a higher risk of incomplete colonoscopy, namely female gender, advanced age, low body mass index, women with a history of hysterectomy, diverticular disease, inflammatory bowel disease, and poor bowel cleansing. ${ }^{13,15}$

Pathology in the lower gastrointestinal tract can be missed by incomplete colonoscopy if additional tests are not performed. Several options are available: re-colonoscopy with a conventional endoscope, computed tomography colonography (CTC), barium enema colonography, or colonoscopy with a double-balloon endoscope. A previous study showed that a secondary colonoscopy after incomplete colonoscopy yielded initially missed advanced neoplasia (CRC or advanced adeno- 
ma) in $4.3 \%$ of patients. ${ }^{16}$

The double-balloon technique allows the endoscope to pass through difficult fixed angles using the overtube balloon as a fixed point in order to avoid pushing maneuvers off the axis of the colonic loop and potentially dangerous stretching. ${ }^{17}$ An additional advantage of the technique is that it makes shortening of long loops easier by fixing both the endoscope and the overtube. ${ }^{17}$

Since 2007, double-balloon endoscopy (DBE) has been available in our center. This study evaluated all patients who underwent colonoscopy with a double-balloon endoscope after previous incomplete conventional colonoscopy. The results included the cecal intubation rate with double-balloon colonoscopy $(\mathrm{DBc})$, the diagnostic rate using $\mathrm{DBc}$ in the unexplored area of the colon, and the complication rate with $\mathrm{DBc}$. In addition, CTC results performed after incomplete colonoscopy were compared with DBc findings.

\section{MATERIALS AND METHODS}

This was a retrospective, observational, single-center study performed in the Catharina Hospital, a tertiary referral center for DBE and DBc in Eindhoven, The Netherlands. The study included all consecutive patients who underwent DBc and CTC after incomplete conventional colonoscopy between October 2007 and September 2014. All indications for DBc were included. DBE ileocolonoscopies via the anal route with the intention to investigate the small bowel were excluded. All reports of CTC performed in the Catharina Hospital in the same time interval were examined. In case of a positive CTC after incomplete colonoscopy, the CTC findings and clinical course were examined in detail, including the results of subsequent examinations ( $\mathrm{DBc}$, sigmoidoscopy, or colonoscopy) or surgery. Data were retrospectively collected from the local hospital information system or from the referring hospitals in case of missing data.

The primary aim of this study was to determine the cecal intubation rate using $\mathrm{DBc}$. Secondary study aims were to determine the diagnostic rate using $\mathrm{DBc}$ in the unexplored part of the colon and the complication rate with $\mathrm{DBc}$, and to compare the results of CTC performed after incomplete colonoscopy with $\mathrm{DBc}$ findings.

\section{Double-balloon colonoscopy}

All DBc procedures were performed using the Fujinon Double-Balloon Enteroscopy System (EN-450T5; Fujinon $\mathrm{GMBH}$, Willich, Germany). The working length of the DBc scope is $200 \mathrm{~cm}$. The outer diameter of this endoscope is 9.4 $\mathrm{mm}$, with a forceps channel diameter of $2.8 \mathrm{~mm}$. All patients who underwent DBE had bowel preparation with polyethylene glycol solution (Moviprep; Norgine Limited, Hengoed, UK). From 2007 until 2012, bowel preparation used 2 L of Moviprep the evening before the examination; in the last 2 years a split-dose preparation was performed using $1 \mathrm{~L}$ of Moviprep the evening before the examination and $1 \mathrm{~L}$ in the morning on the day of examination. DBc was carried out in patients under conscious sedation, which was achieved with intravenous midazolam and fentanyl.

All DBcs were performed by one of two experienced double-balloon endoscopists in our department. The findings, including location in the colon, interventions, and complications were carefully recorded in the endoscopy report and digital patient file. An advanced adenoma was defined as a large polyp (>10 mm) or a polyp with high-grade dysplastic lesions. The location of the abnormality was determined by the insertion depth of the endoscope and clinical landmarks (e.g., the appendiceal orifice, Bauhin's Valve, hepatic or splenic flexure, and triangular lumen in the transverse colon). Cecal intubation was defined as visualization of the appendiceal orifice. In some patients, one or more interventions were performed during DBc, e.g., biopsy, argon plasma coagulation, clipping, or dilation.

\section{Computed tomography colonography}

Since 2009, CTC has been performed in our center as an alternative to colonoscopy, and includes a non-enhanced scan in the supine and prone position. For a dedicated CTC exam, bowel preparation consists of low-fiber diet the day before the exam combined with $50 \mathrm{~mL}$ oral contrast medium for all 3 meals, and a liquid breakfast on the day of the exam. Before the exam, $50 \mathrm{~mL}$ of oral contrast is given for optimal fecal tagging. All colonic and extracolonic findings are evaluated and classified according to a CTC Reporting and Data System. ${ }^{18}$

In case of incomplete colonoscopy, the patient undergoes CTC on the same day after receiving $100 \mathrm{~mL}$ of oral contrast medium at least $1 \mathrm{~h}$ prior to the exam. No ingestion of food is allowed between the incomplete colonoscopy and the CTC. In our institution Telebrix Gastro ${ }^{\circledR}$ (Guerbet, Aulnay-sous-Bois, France) is the contrast medium of choice.

\section{Data analysis}

Continuous variables were presented as mean with standard deviation or median with range, depending on normality. Categorical variables were presented as frequencies and percentages. Differences were evaluated using the chi-square test or Fisher's exact test in case of small numbers. $P<0.05$ was considered statistically significant.

All data were analyzed with Statistical Package for Social Science (SPSS) software version 21.0 (SPSS Inc., Chicago, IL, USA). 


\section{RESULTS}

Between October 2007 and September 2014 a total of 63 DBcs were performed after incomplete routine colonoscopy in a total of 61 patients. Baseline characteristics of these patients are presented in Table 1. Indications for original colonoscopy

Table 1. Characteristics of Patients that Underwent Double-Balloon Colonoscopy after Incomplete Colonoscopy ( $n=61$ patients and $63 \mathrm{DBc}$ procedures)

\begin{tabular}{|c|c|}
\hline & $\begin{array}{l}\text { Patients that under- } \\
\text { went } \mathrm{DBc}(n=61)\end{array}$ \\
\hline Mean age (range) (yr) & $65(29-82)$ \\
\hline Men/Women, $n(\%)$ & $34 / 27(56 \% / 44 \%)$ \\
\hline Previous abdominal surgery, $n(\%)$ & $24(39 \%)$ \\
\hline Indication for total colonoscopy, $n(\%)$ & $n=63 \mathrm{DBc}$ \\
\hline $\begin{array}{l}\text { Screening or surveillance for adenomas } \\
\text { or carcinoma }\end{array}$ & $47(74 \%)$ \\
\hline Abdominal pain & $8(13 \%)$ \\
\hline Diarrhea & $2(3 \%)$ \\
\hline Macroscopic blood loss & $3(5 \%)$ \\
\hline Occult blood loss or iron deficient anemia & $3(5 \%)$ \\
\hline Extent of incomplete colonoscopy, $n(\%)$ & $n=63 \mathrm{DBc}$ \\
\hline Rectosigmoid & $2(3 \%)$ \\
\hline Colon descendens & $3(4 \%)$ \\
\hline Colon transversum & $40(63 \%)$ \\
\hline Colon ascendens & $18(30 \%)$ \\
\hline Reasons for incomplete colonoscopy, $n(\%)$ & $n=63 \mathrm{DBc}$ \\
\hline Dolichocolon & $41(65 \%)$ \\
\hline Looping & $13(21 \%)$ \\
\hline Dolichocolon and looping & $5(8 \%)$ \\
\hline Adhesions & $4(6 \%)$ \\
\hline
\end{tabular}

$\mathrm{DBc}$, double-balloon colonoscopy.

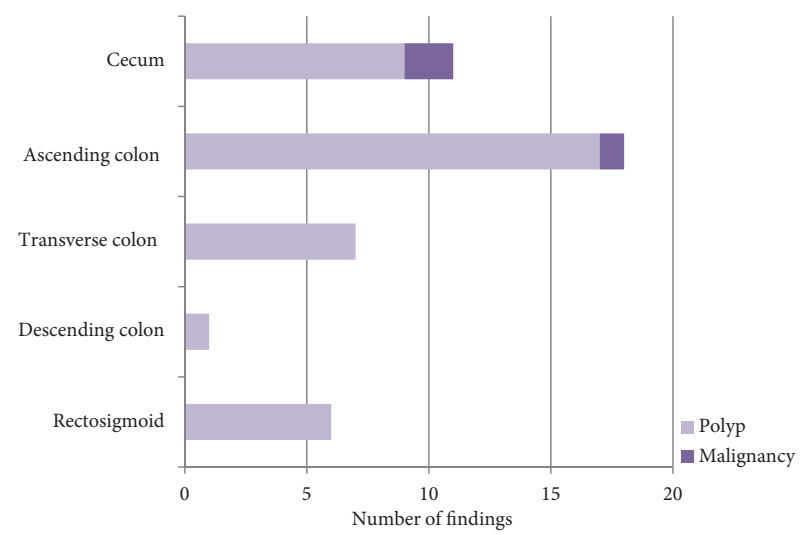

Fig. 1. Findings in double balloon colonoscopy after incomplete colonoscopy ( $n=63$ procedures) in the unexplored part of the colon. and follow-up DBc were screening or surveillance for adenoma or carcinoma (74\%), abdominal pain (13\%), diarrhea (3\%), macroscopic blood loss (5\%), and occult blood loss or iron-deficiency anemia (5\%). Colonoscopy failure was because of dolichocolon in 41 procedures (65\%), looping of the endoscope in $13(21 \%)$, combined dolichocolon and looping in 5 (8\%), and colon fixation because of adhesions in 4 (6\%).

$\mathrm{DBc}$ cecal intubation rate was $95 \%$. Two incomplete procedures (both indicated for screening or surveillance for adenoma or carcinoma) were caused by inadequate colon cleansing. One of these patients underwent laparoscopy afterwards for persistent ileus, and an obstructive adenocarcinoma was found in the ascending colon. The other patient underwent successful second DBc after extra bowel cleansing and an adenoma was found in the ascending colon. Looping of the endoscope caused incomplete $\mathrm{DBc}$ in one patient. The indication for this $\mathrm{DBc}$ was abdominal pain. CT of the colon showed no abnormalities in this patient.

The detection rate of polyps or (suspected) carcinomas using $\mathrm{DBc}$ in the previously unexplored part of the colon was $58 \%$. A carcinoma was found in 3 procedures (5\%), high-grade dysplastic adenoma in 2 (3\%), and sessile serrated adenoma in $2(3 \%)$, as shown in Fig. 1. Two or more polyps were seen in 26 procedures (41\%). Table 2 presents the main diagnosis in the previously uninvestigated parts of the colon, reached using DBc. According to indication, the detection rate was $68 \%$ for screening or surveillance for adenoma or carcinoma (32/47), $13 \%$ for abdominal pain $(1 / 8), 0 \%$ for diarrhea $(0 / 2), 67 \%$ for macroscopic blood loss (2/3), and $67 \%$ for occult blood loss or iron-deficiency anemia $(2 / 3)$.

Diagnostic biopsy was taken in 16\% of DBE procedures, revealing carcinoma $(n=3)$, sessile serrated adenoma $(n=1)$, nonspecific chronic inflammation $(n=1)$, or no abnormality $(n=5)$. An intervention was performed in 38 of 63 procedures (60\%), i.e., 35 polypectomies and 3 cases of argon plasma coagulation. Argon plasma coagulation was used to remove small residual adenomatous spots and/or coagulate small vessels to prevent

Table 2. Main Diagnosis Found during Double-Balloon Colonoscopy after Incomplete Colonoscopy ( $n=63$ procedures)

\begin{tabular}{lcl}
\hline Diagnosis & $\boldsymbol{n ( \% )}$ & $\begin{array}{c}\text { Therapeutic } \\
\text { consequences }\end{array}$ \\
\hline No diagnosis & $26(41 \%)$ & - \\
Carcinoma & $3(5 \%)$ & Surgical resection \\
\hline Adenoma(s) & & \\
\hline Low grade dysplasia & $20(32 \%)$ & Endoscopic resection \\
\hline High grade dysplasia & $2(3 \%)$ & Endoscopic resection \\
\hline Sessile serrated & $2(3 \%)$ & Endoscopic resection \\
\hline Hyperplastic polyp(s) & $10(16 \%)$ & Endoscopic resection \\
\hline
\end{tabular}


Table 3. Findings of Computed Tomography Colonography in Patients with Prior Incomplete Colonoscopy ( $n=213$ procedures) and Subsequent Diagnostic Steps with Eventual Results

\begin{tabular}{|c|c|c|c|}
\hline Findings diagnostic CTC & $n(\%)$ & Subsequent examinations or surgery & Subsequent findings \\
\hline No abnormalities in colon & $139(65 \%)$ & - & - \\
\hline \multirow[t]{2}{*}{ Suspicion of colon carcinoma } & $6(3 \%)$ & Surgical resection (4) & $\begin{array}{l}\text { Adenocarcinoma (2) } \\
\text { Diverticulitis (2) }\end{array}$ \\
\hline & & $\begin{array}{l}\text { Repeat endoscopy } \\
\text { - Colonoscopy (1) } \\
\text { - Sigmoidoscopy (1) }\end{array}$ & $\begin{array}{l}\text { Adenocarcinoma (1) } \\
\text { Diverticulitis (1) }\end{array}$ \\
\hline \multirow[t]{3}{*}{ Aspecific wall thickness } & $13(6 \%)$ & Surgical resection (1) & Diverticulitis (1) \\
\hline & & $\begin{array}{l}\text { Repeat endoscopy } \\
\text { - Colonoscopy (2) } \\
\text { - Sigmoidoscopy (1) }\end{array}$ & Diverticulitis (3) \\
\hline & & \multicolumn{2}{|l|}{ No action (9) } \\
\hline Polypoid lesion & $55(26 \%)$ & $\begin{array}{l}\text { Repeat endoscopy } \\
\text { - DBc (19) } \\
\text { - Colonoscopy (16) } \\
\text { - Sigmoidoscopy (4) }\end{array}$ & $\begin{array}{l}\text { Adenoma (20) } \\
\text { Adenocarcinoma (1) } \\
\text { Hyperplastic lesion (1) } \\
\text { Lipoma (1) } \\
\text { No lesion confirmed (16) }\end{array}$ \\
\hline \multicolumn{4}{|c|}{ No action (16) } \\
\hline
\end{tabular}

CTC, computed tomography colonography; DBc, double-balloon colonoscopy.

post-polypectomy bleeding in these 3 procedures

In the $63 \mathrm{DBc}$ procedures, no minor and only 1 major complication (1.5\%) occurred, i.e., arterial bleeding in the ascending colon immediately after adenomatous polyp removal (low-grade dysplasia, $14 \mathrm{~mm}$ ). Adrenalin injections and clipping were not effective, finally resulting in a right hemicolectomy, which was performed without complications.

The procedure time of $\mathrm{DBc}$ was not precisely monitored during the study period. However, all DBc procedures were planned for a maximum duration of $1 \mathrm{~h}$; most procedures were performed within $45 \mathrm{~min}$.

Thirty-four DBcs were preceded by CTC (54\%). Two CTCs were performed in another hospital. Twenty-one (62\%) DBcs confirmed the prior CTC diagnosis and 13 (38\%) could not.

During the study period, 760 CTCs were performed in our center for several indications. The results of all 213 CTCs after prior incomplete conventional colonoscopy were divided into 4 groups: no abnormalities (139), suspicion of a malignant lesion (6), nonspecific wall thickening (13), and polypoid lesion (55) (Table 3). In the absence of abnormalities, no follow-up endoscopy was performed. In the group with suspected malignant lesions, surgery was performed in 4 cases and repeat endoscopy in 2. Three adenocarcinomas of the colon and 1 case of diverticulitis were found in resected specimens. Repeat endoscopy confirmed carcinoma in 1 case and diverticulitis in the other. Overall CTC diagnosis was correct in $67 \%$ of these suspected cases. Polypoid lesions found in CTC were confirmed using re-endoscopy in 23 cases. Re-endoscopy found 20 adenomas with low-grade dysplasia, 1 hyperplastic lesion, 1 lipoma, and 1 malignant polyp. No polyp was found with re-endoscopy in 16 cases, and no repeat endoscopy was performed in 16 cases. Of 19 re-endoscopies performed with $\mathrm{DBc}, 17$ confirmed CTC findings. Nonspecific wall thickening of the colon was seen in 13 CTCs. Subsequent endoscopic analysis showed no malignancy, but 4 cases of diverticulitis were found in these patients.

In 33 patients, no further examination was performed after CTC with a positive finding for several reasons: age, patient condition, location of a possible lesion in the previously examined part of the colon, or clinical signs of diverticulitis.

The positive predictive value of CTC for clinically significant lesions (malignancy, polyp, diverticulitis) was 65\%. This calculation included only patients with follow-up endoscopy or surgery. In the 6 patients with a strong suspicion of malignancy on CTC, 4 had a malignant lesion (67\%); the other 2 were diagnosed with diverticulitis. A negative predictive value could not be determined because a negative CTC was not followed by subsequent examinations in this retrospective study design.

\section{DISCUSSION}

This retrospective observational study of DBE procedures of the colon $(\mathrm{DBc})$ after incomplete colonoscopy showed successful cecal intubation in the vast majority of cases (95\%). 
More importantly, it demonstrated a pathology detection rate of $58 \%$ in the previously unexplored part of the colon, and revealed 5\% carcinoma rate and another $6 \%$ advanced adenoma rate (large polyps or high-grade dysplastic lesions).

The DBc cecal intubation rate of $95 \%$ is comparable to that in other studies, which reported success rates of $88.6 \%$ $100 \%{ }^{11,12,17,19-23}$ Two of the 3 incomplete DBcs were because of inadequate cleansing of the bowel, which should be avoidable in future to improve intubation rates.

Various alternative techniques are available to complete colon inspection after previous incomplete colonoscopy, e.g., the use of a gastroscope, ${ }^{24}$ push enteroscopy, ${ }^{25}$ a variable-stiffness colonoscope, ${ }^{26}$ or a pediatric colonoscope, ${ }^{27,28}$ which showed a somewhat lower average cecal intubation success rates ${ }^{24-28}$ compared to balloon-assisted endoscopy. $\operatorname{Rex}^{14}$ stated that the reason for previous incomplete colonoscopy determines the optimal choice of endoscope in a subsequent procedure. In case of dolichocolon or looping, $\mathrm{DBc}$ or push enteroscopy with an overtube is recommended, whereas a pediatric colonoscope is indicated for a difficult sigmoid, e.g., with diverticulosis/diverticulitis or adhesions, because of the smaller diameter of the endoscope. Gawron et al. ${ }^{29}$ showed a high cecal intubation rate of $96 \%$ with use of standard endoscopes, mostly in patients with a looping or tortuous colon, whereas patients with a dolichocolon needed other endoscopes more frequently. Our study showed no differences in cecal intubation success rates among reasons for previous incomplete colonoscopy; thus, $\mathrm{DBc}$ appears useful for all difficult colon types. CTC is often used as an alternative to visualize the colon. The advantages of CTC are high diagnostic performance, ${ }^{30}$ less-invasive imaging technique, no need for sedation, short recovery time, and little patient discomfort. Another advantage is the detection of extracolonic findings. In patients with a positive immunochemical fecal blood test (iFOBT), CTC is also considered an adequate option because of a sensitivity of $88.8 \%$, as shown by a major systematic review and meta-analysis by Plumb et al. in 2014 . $^{31}$

This study also demonstrated that $\mathrm{DBc}$ is a safe procedure: only 1 patient had a major complication, i.e., arterial bleeding due to polypectomy with a need for right hemicolectomy because of failure of conservative therapies. Most studies reported no or few major complications. ${ }^{21-23}$ Another Dutch clinical study reported a complication rate of $1.8 \%$, consisting of 2 cases of arterial bleeding. ${ }^{12}$

The most important limitation of this study is its retrospective nature. In addition, the $\mathrm{DBc}$ detection rate may have been influenced by selection bias, as some patients had CTC prior to DBc. However, the patients who did not have CTC prior to $\mathrm{DBc}$ had a similar detection rate and CTC results were not confirmed in $38 \%$. Moreover, no subsequent control exam- ination was performed in case of negative $\mathrm{DBc}$ or CTC. The procedure times for $\mathrm{DBc}$ were not precisely recorded, but all were performed within $60 \mathrm{~min}$. Nevertheless, this study from an experienced center performing DBc as well as CTC shows important clinical findings that are applicable in daily clinical decision-making in case of incomplete colonoscopy.

The choice of examination following incomplete colonoscopy is difficult. We advise use of $\mathrm{DBc}$ in patients with a high likelihood of an abnormality and need for intervention. In this study, patients who underwent DBc for screening or surveillance for adenoma or carcinoma, macroscopic blood loss, and occult blood loss or iron-deficiency anemia had the highest detection rate $(67 \%-68 \%)$. Patients with a positive iFOBT in the national screening program for CRC have a similar risk. The invasiveness of $\mathrm{DBc}$ is a point mentioned frequently as a disadvantage, but we think the invasiveness is similar to that of colonoscopy, and is therefore feasible in patients who underwent previous colonoscopy. A disadvantage of $\mathrm{DBc}$ is the availability, whereas CTC is available in more places. The clinical predictors that can determine which examination is best after incomplete colonoscopy require further investigation.

To conclude, $\mathrm{DBc}$ is safe and effective for complete inspection of the entire colon in difficult cases in which conventional colonoscopy failed. Because of the high detection rate, possibility of intervention in $\mathrm{DBc}$, and false-positive findings in CTC, DBc is preferred over CTC, especially in patients with a high likelihood of abnormalities, such as those identified via colorectal screening programs.

Conflicts of Interest

The authors have no financial conflicts of interest.

\section{REFERENCES}

1. Micksche M, Lynge E, Diehl V, et al. [Recommendations on cancer screening in the European Union]. Bull Cancer 2001;88:687-692.

2. Rex DK, Petrini JL, Baron TH, et al. Quality indicators for colonoscopy. Am J Gastroenterol 2006;101:873-885.

3. Pohl H, Robertson DJ. Colorectal cancers detected after colonoscopy frequently result from missed lesions. Clin Gastroenterol Hepatol 2010;8:858-864.

4. Winawer SJ, Zauber AG, Ho MN, et al. Prevention of colorectal cancer by colonoscopic polypectomy. The national polyp study workgroup. $\mathrm{N}$ Engl J Med 1993;329:1977-1981.

5. Zauber AG, Winawer SJ, O'Brien MJ, et al. Colonoscopic polypectomy and long-term prevention of colorectal-cancer deaths. N Engl J Med 2012;366:687-696.

6. Baxter NN, Goldwasser MA, Paszat LF, Saskin R, Urbach DR, Rabeneck L. Association of colonoscopy and death from colorectal cancer. Ann Intern Med 2009;150:1-8.

7. Marshall JB, Barthel JS. The frequency of total colonoscopy and terminal ileal intubation in the 1990s. Gastrointest Endosc 1993;39:518-520.

8. Dafnis G, Granath F, Påhlman L, Hannuksela H, Ekbom A, Blomqvist P. The impact of endoscopists' experience and learning curves and in- 
terendoscopist variation on colonoscopy completion rates. Endoscopy 2001;33:511-517.

9. Jung Y, Lee SH. How do I overcome difficulties in insertion? Clin Endosc 2012;45:278-281.

10. Hotta K, Katsuki S, Ohata K, et al. Efficacy and safety of endoscopic interventions using the short double-balloon endoscope in patients after incomplete colonoscopy. Dig Endosc 2015;27:95-98.

11. Hotta K, Katsuki S, Ohata K, et al. A multicenter, prospective trial of total colonoscopy using a short double-balloon endoscope in patients with previous incomplete colonoscopy. Gastrointest Endosc 2012;75:813-818.

12. Becx MC, Al-Toma A. Double-balloon endoscopy: an effective rescue procedure after incomplete conventional colonoscopy. Eur J Gastroenterol Hepatol 2014;26:519-522.

13. Koido S, Ohkusa T, Nakae K, et al. Factors associated with incomplete colonoscopy at a Japanese academic hospital. World J Gastroenterol 2014;20:6961-6967.

14. Rex DK. Achieving cecal intubation in the very difficult colon. Gastrointest Endosc 2008;67:938-944.

15. Pullens HJ, Siersema PD. Quality indicators for colonoscopy: current insights and caveats. World J Gastrointest Endosc 2014;6:571-583.

16. Neerincx M, Terhaar sive Droste JS, Mulder CJ, et al. Colonic workup after incomplete colonoscopy: significant new findings during follow-up. Endoscopy 2010;42:730-735.

17. Gay G, Delvaux M. Double-balloon colonoscopy after failed conventional colonoscopy: a pilot series with a new instrument. Endoscopy 2007;39:788-792.

18. Pooler BD, Kim DH, Lam VP, Burnside ES, Pickhardt PJ. CT colonography reporting and data system (C-RADS): benchmark values from a clinical screening program. AJR Am J Roentgenol 2014;202:1232-1237.

19. Yamada A, Watabe $\mathrm{H}$, Takano N, et al. Utility of single and double balloon endoscopy in patients with difficult colonoscopy: a randomized controlled trial. World J Gastroenterol 2013;19:4732-4736.

20. Moreels TG, Macken EJ, Roth B, Van Outryve MJ, Pelckmans PA. Cecal intubation rate with the double-balloon endoscope after incomplete conventional colonoscopy: a study in 45 patients. J Gastroenterol Hepatol 2010;25:80-83.
21. Kaltenbach T, Soetikno R, Friedland S. Use of a double balloon enteroscope facilitates caecal intubation after incomplete colonoscopy with a standard colonoscope. Dig Liver Dis 2006;38:921-925.

22. Dzeletovic I, Harrison ME, Pasha SF, et al. Comparison of single- versus double-balloon assisted-colonoscopy for colon examination after previous incomplete standard colonoscopy. Dig Dis Sci 2012;57:2680-2686.

23. Pasha SF, Harrison ME, Das A, Corrado CM, Arnell KN, Leighton JA. Utility of double-balloon colonoscopy for completion of colon examination after incomplete colonoscopy with conventional colonoscope. Gastrointest Endosc 2007;65:848-853.

24. Paonessa NJ, Rosen L, Stasik JJ. Using the gastroscope for incomplete colonoscopy. Dis Colon Rectum 2005;48:851-854.

25. Lichtenstein GR, Park PD, Long WB, Ginsberg GG, Kochman ML. Use of a push enteroscope improves ability to perform total colonoscopy in previously unsuccessful attempts at colonoscopy in adult patients. Am J Gastroenterol 1999;94:187-190.

26. Shumaker DA, Zaman A, Katon RM. Use of a variable-stiffness colonoscope allows completion of colonoscopy after failure with the standard adult colonoscope. Endoscopy 2002;34:711-714.

27. Marshall JB. Use of a pediatric colonoscope improves the success of total colonoscopy in selected adult patients. Gastrointest Endosc 1996;44:675678.

28. Saifuddin T, Trivedi M, King PD, Madsen R, Marshall JB. Usefulness of a pediatric colonoscope for colonoscopy in adults. Gastrointest Endosc 2000;51:314-317.

29. Gawron AJ, Veerappan A, Keswani RN. High success rate of repeat colonoscopy with standard endoscopes in patients referred for prior incomplete colonoscopy. BMC Gastroenterol 2014;14:56.

30. Pickhardt PJ, Hassan C, Halligan S, Marmo R. Colorectal cancer: CT colonography and colonoscopy for detection--systematic review and meta-analysis. Radiology 2011;259:393-405.

31. Plumb AA, Halligan S, Pendsé DA, Taylor SA, Mallett S. Sensitivity and specificity of CT colonography for the detection of colonic neoplasia after positive faecal occult blood testing: systematic review and meta-analysis. Eur Radiol 2014;24:1049-1058. 